Review Article

\title{
Discovering the Use of Complementary and Alternative Medicine in Oncology Patients: A Systematic Literature Review
}

\author{
Fatmah Alsharif $(\mathbb{D}$ \\ Medical Surgical Nursing Department, School of Nursing, King Abdulaziz University, Jeddah, Saudi Arabia \\ Correspondence should be addressed to Fatmah Alsharif; falsharif@kau.edu.sa
}

Received 1 December 2020; Revised 16 December 2020; Accepted 22 December 2020; Published 13 January 2021

Academic Editor: Muhammad Nabeel Ghayur

Copyright (C) 2021 Fatmah Alsharif. This is an open access article distributed under the Creative Commons Attribution License, which permits unrestricted use, distribution, and reproduction in any medium, provided the original work is properly cited.

\begin{abstract}
Background. Patients with cancer pursue all possible opportunities of effective remedies. In Saudi Arabia, most patients have tried complementary medicine during their cancer treatment plan; however, some complementary medicines might pose a danger to health. In Arab countries, studies regarding the use of complementary medicines and the intentions behind using complementary medicines among cancer patients are inadequate and all but nonexistent. The aim of this review was to focus on demographic, prevalence, and reasons for complementary and alternative medicine use among patients with cancer. Methods. A rigorous literature search was conducted for articles published in the English language, using the search terms "complementary and alternative medicine," "oncology," "malignancy" AND "cancer patients" in five scientific databases, namely, Medical Literature On-Line (MEDLINE), PubMed, Web of Science, Psychology Information (PsycINFO), and Google Scholar, from 2010 to 2020. Results. Of the 13,160 studies returned from the search, only 12 were eligible and included in this review. The combined prevalence for using complementary and alternative medicines across all studies totaled $25 \%-80 \%$ of cancer patients for the treatment of their cancers. Natural products, including vitamins and minerals, herbal products, and relaxation, were the most common type of complementary and alternative medicines used. The reason for using complementary and alternative medicines was reported to be their helpfulness in recovering, healing, and improving health. Most of the studies mentioned that participants obtained their complementary and alternative medicines from multiple sources, including the media, family and friends, and physicians. Conclusion. The use of complementary and alternative medicines in cancer patients can be inferred as an attempt to investigate all possibilities, a manifestation of a coping style, or an illustration of unmet desires in the cancer management continuum. Anyhow, there should be adequate communication between healthcare providers and patients, which is crucial for establishing a trusting healthcare provider-patient relationship. Relevance to clinical practice. It is crucial that healthcare providers explore the use of complementary and alternative medicines with their cancer patients, as well as educating them about the possible usefulness of therapies based on the available evidence.
\end{abstract}

\section{Introduction}

Cancer is deliberated as a common health problem, associated with significant disabilities, and is one of the three leading causes of death worldwide [1]. According to the World Health Organization (WHO), it is estimated that cancer rates will be increased by $50 \%$ to 15 million by 2030 . Thus, most cancer patients and their families seek all possible options related to effective treatment to manage the trauma of distress, worries, and immense fear associated with their condition $[2,3]$.

Some patients explore complementary and alternative medicine (CAM) choices, which constitute a type of medicine independent of conventional medicine. This practice is widely used across the world and can be explained as any usage that is not part of conventional medicine [4]. It is commonly the application of the sum of knowledge and practices developed through experiences and beliefs of distinctive cultures [1].

Numerous patients that feel worried decide to leave their course of conventional medicine and instead seek help via complementary and alternative medicines, which are therefore gaining increasing interest, especially in the field of oncology [5]. Due to this trend, patients' interests should be given due deliberation in current oncology practices and 
should be addressed in such a way as to provide recommendations regarding acceptable and individualized therapy merged with supportive care, including CAMs, under standardized oncology protocols [6]. However, the data pertaining to CAMs and their inappropriate management are a challenge; for example, herbal supplies, which are considered a complementary medicine, may interact with conventional treatments, potentially exposing the patient to an increased risk of ill health. Moreover, CAMs are potentially subject to corruption, contamination, or replacement with other more harmful products [7]. The literature of this study aimed to summarize the use of CAMs among patients with cancer. This review involved the following main phases: data search strategy, article selection, data extraction, data analysis/synthesis, and critical appraisal of the included articles [8]. The particular questions about patients with cancer were as follows [2]: Which types of CAM were stated [9]? What were the sources of information about CAM [4]? What factors contributed to CAM use? [7] Why did patients decide to use CAM?

\section{Methodology}

Systematic reviews promote a present basis for any observation and might broaden new study ideas. At the beginning of a written document, it allows readers to enjoy and become expertise in the contemporary tendencies of troubles and clears up the importance of revolutionary studies [10]. Tappen (2016) demonstrated that literature reviews involve methodically determining, finding, and examining materials that are associated with the study question [8]. The literature of this study aimed to discuss the use of CAMs among patients with cancer. This review involved the following main phases: data search strategy, article selection, data extraction, data analysis/synthesis, and critical appraisal of the included articles [8].

2.1. Data Search Strategy. This study was carried out by analyzing the literature through PRISMA's evidence-based data evaluation search strategy. It illustrates the reporting of randomized study assessment evaluations, although it can also be used as a framework for reporting systematic reviews of other types of research.

In order to extract the data for the factors highlighted, through the systematic use of electronic databases, the investigator collected the appropriate published papers and articles. Nonetheless, a number of the foremost inclusive journal places that used to cognizance the literature in the field of CAM research are Medical Literature On-Line (MEDLINE), PubMed, Web of Science, Psychology Information (PsycINFO), and Google Scholarreplace.

The following keywords were applied for the electronic search: "Complementary medicine," "alternative medicine," "oncology," "malignancy," "cancer," and "cancer patients." These keywords were used in combination or separately. Furthermore, the reference sections of the relevant articles were checked to distinguish additional trials unexploited by the electronic search. There were some digital databases that confirmed obsolete in attempting to find the associated articles via keywordsreplace.

The selected electronic records were chosen on the basis of the extensive range of disciplines they covered and their integrity, in addition to those almost certainly about issues relevant to Saudi Arabia.

2.2. Article Selection and Data Extraction. The articles were selected based on predefined inclusion and exclusion criteria. The research articles and journals were examined critically through and extracted from online computerized search engines. The inclusion criteria for the research articles were as follows:

(1) Published in English language only

(2) A systematic search of peer-reviewed, published literature from 2010 to 2020 conducted between March and August 2020

(3) Focusing on the CAMs used by cancer patients

(4) Relying on both qualitative and quantitative evidence or on mixed research methodsreplace

(5) Including a list of references of all reviewed articles with an appropriate inclusion quality, and with no follow-up by the investigators to extract further primary or secondary data

Meanwhile, articles were excluded if

(1) they were unpublished articles or studies

(2) they were opinions or commentaries

(3) they were not published in the English language

Primarily, the titles and abstracts of relevant articles recognized during the electronic database search were scrutinized and included or excluded based on the predefined selection criteria listed above. The population, intervention, comparison, outcomes, and study design parameters were used to describe the eligibility criteria.

The data pertaining to the objectives, sample population, design, methodology, and data collection procedures were extracted from the selected articles. In addition, findings, discussions, and conclusions were also analyzed in order to verify the relationship among some of the variables. The variables that were searched were those related to CAM use in cancer patients. All the data collected are clustered, summarized and compared for analysis in the studies.

2.3. Data Analysis and Synthesis. In order to thoroughly reflect on the literature reviews, the research reviewed andanalysis several relevant studies within the 2010-2020 period. Some of the studies highlighted and addressed Some common problems that countries might have come to know during catastrophic situations in which an extensive number of fully competent nurses would have been required. In order to demonstrate these points, the researchers made significant use of all current journal articles, reports, editorials, and correlational descriptive studies. In order to better understand the point of view of nurses on their lack of 
expertise in delivering good services, the study also provided a keen emphasis on both primary and secondary research papers.

2.4. Critical Appraisal of the Included Studies. The included studies were critically appraised; they were evaluated for their psychometric measures such as reliability and validity, the two necessary features that determine the quality of a quantitative study. The reliability of quantitative research is correlated with its consistency, stability, agreement, reproducibility, repeatability, and homogeneity. Nevertheless, validity refers to how well-founded and accurate an instrument or a study measure is [10].

The citations used in this research paper have been correctly cited and cover the key topic-related primary headings. Approximately 600 articles and papers that were not in accordance with the study purpose were also omitted by the investigator.

\section{Results}

From the database, 13,160 articles (2010-2020) were identified, of which 12 met the criteria for inclusion. The documents were original quantitative research articles, published in English and local languages, and directly relevant to the aim of the study (Table 1). Four of the studies (33.3\%) were conducted in Asia, followed by three studies (25\%) in Arab countries, two studies (16.7\%) in Canada, two $(16.7 \%)$ in Europe, and one $(8.3 \%)$ in South America (Figure 1).

3.1. Sociodemographic Factors. The sociodemographic factors found to be correlated with the practice of CAM enclosed age, education level, income, marital status, and presence of a support group. Most of the samples in the studies were females, aged over 40 years and married, and had moderate level of income $[1,2,4,11-13]$. Other studies reported on middle-aged males with a high income (Table 1) $[9,14]$.

Out of the 12 studies that investigated sociodemographic factors and the use of CAMs in cancer patients, six reported that younger female patients with a moderate education level were more likely to use CAMs than those who were male, older, and had a lower education level $[1,2,9,12,13,15]$. Three studies reported that males were more likely to use CAMs than females $[5,11,14]$. Only a few studies found that age and education were not related to the use of CAMs in cancer patients $[4,7,16]$.

Of the 12 studies, eight reported that cancer patients who had a higher income were more likely to use CAMs than those who had a lower income $[1,7,9,12,16]$. Meanwhile, two studies reported that those with low income were more likely to use CAMs than those who had a higher income $[2,4,15]$, and other study found a similar relationship between low and moderate incomes (rather than a high income) [13]. However, only one study reported a lack of a relationship between income and CAM use [11].
Regarding marital status, seven studies revealed that married patients were more likely to use CAMs than those who were unmarried $[1,2,7,9,12,13,16]$; however, three studies showed no relationship between marital status and CAM use (Table 1) $[5,11,14]$.

\subsection{Types of Complementary and Alternative Medicines Used.} Five studies mentioned that natural products, including minerals, vitamins, and relaxation, were the common types of CAMs used [1, 2, 4, 7, 16]. Four studies reported that unlabeled fresh and processed herbal products and honey were the most commonly used complementary medicines. Porcupine flower, lingzhi, Ephedra foeminea (Alanda) $[2,4,11,14]$, Chinese medicine, reflexology, and hypnosis were also reported to be common [5]. One study mentioned that cancer patients also used traditional medicines [15]. Two studies conducted in Arab countries (Tunisia and Egypt) mentioned that cancer patients' use of CAMs was religious-based $[2,11]$, while in a study conducted in Saudi Arabia, most of the cancer patients used Quran recitation, supplication, Zamzam water, olive oil, and black seeds (Nigella sativa) [9].

Among the reports, the reported incidence of use of each item varied. In general, the most prevalent natural commodity in use was natural merchandise containing various plant parts prepared in various ways. Only two of the included studies did not report the forms of CAMs used by their study participants (Table 1) [12].

\subsection{Reasons for Using Complementary and Alternative} Medicines. The reasons for using of CAMs reported by cancer patients were diverse, and some reported more than one reason. All 12 studies reported that patients used complementary and alternative medicines because they believed it was helpful to their recovering and healing and improved their health $[1,2,4,5,7,9,11-16]$.

The other reasons included the enhancement of physical and emotional well-being, as well as rising their body's capability to fight cancer $[1,2,5,9,14]$, strengthening their immune system $[4,5,9,12]$, following their physician's suggestions [4], controlling their pain, and improving their appetite $[9,15]$. CAMs were predominantly used to prevent or treat the side effects of anticancer treatments $[5,15]$. One study did not mention any specific reasons, but the participants said they were just trying to do everything that could help them [16].

Additionally, the results of some of the studies showed that the overall degree of satisfaction regarding complementary and alternative medicine usage was generally high $[5,9,11,15]$, while one study showed low satisfaction of the use of complementary and alternative medicines [13].

3.4. Source of Information. Most of the studies mentioned that patients obtained information about CAMs from multiple sources. Internet (media) and the social network Facebook were the main sources of information regarding CAMs, followed by family and friends $[7,9,11,14,16]$. The 


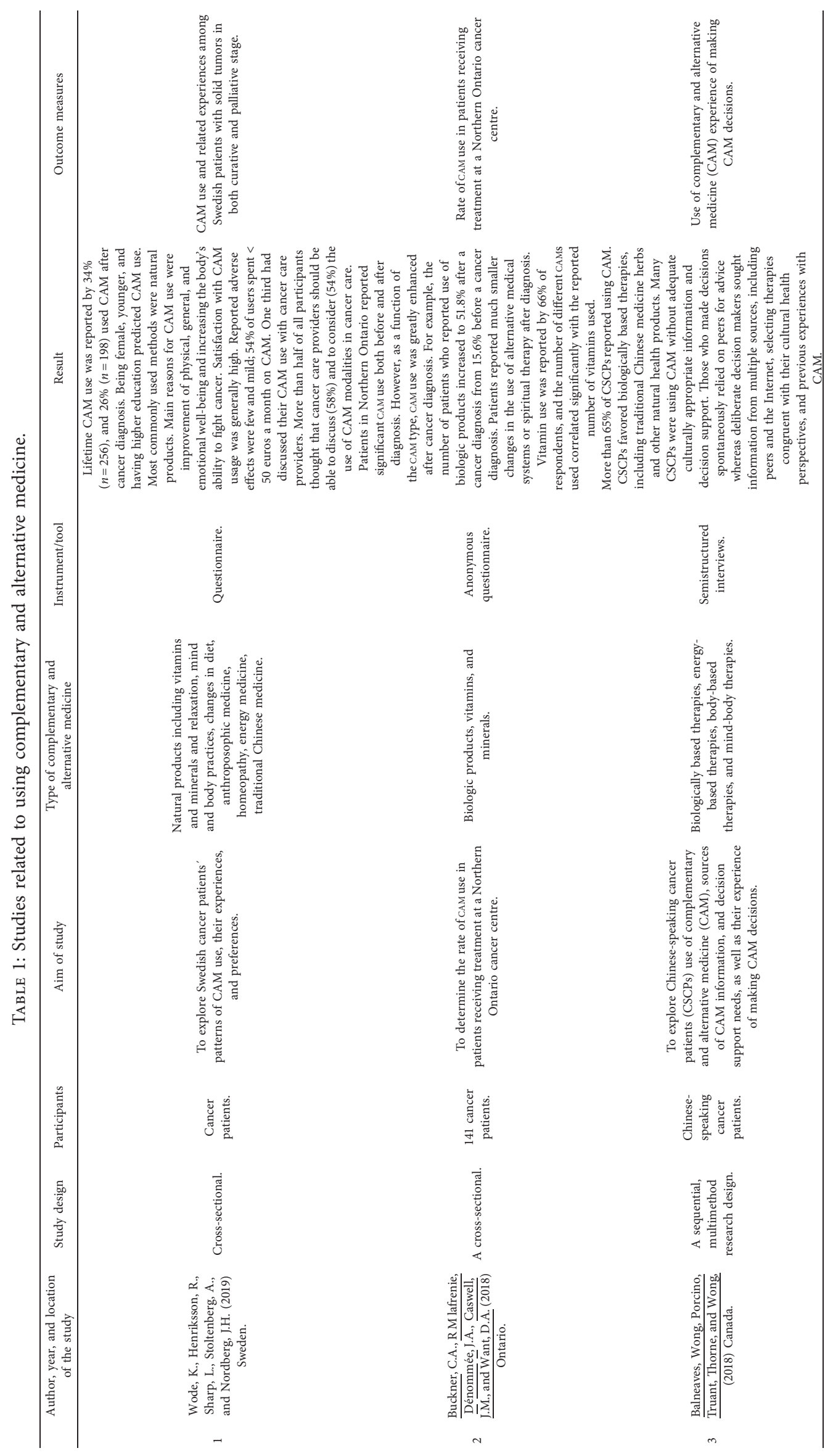




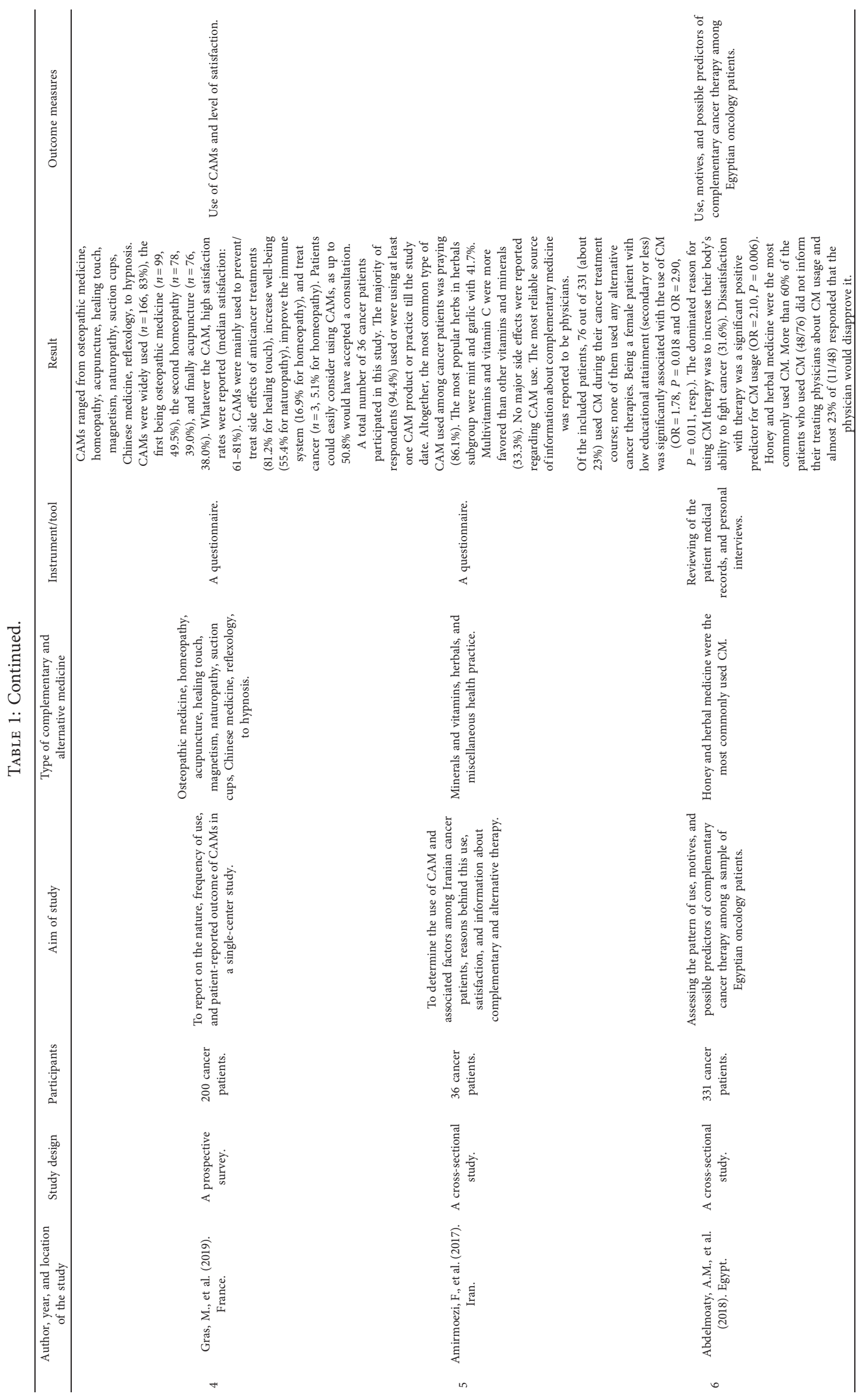




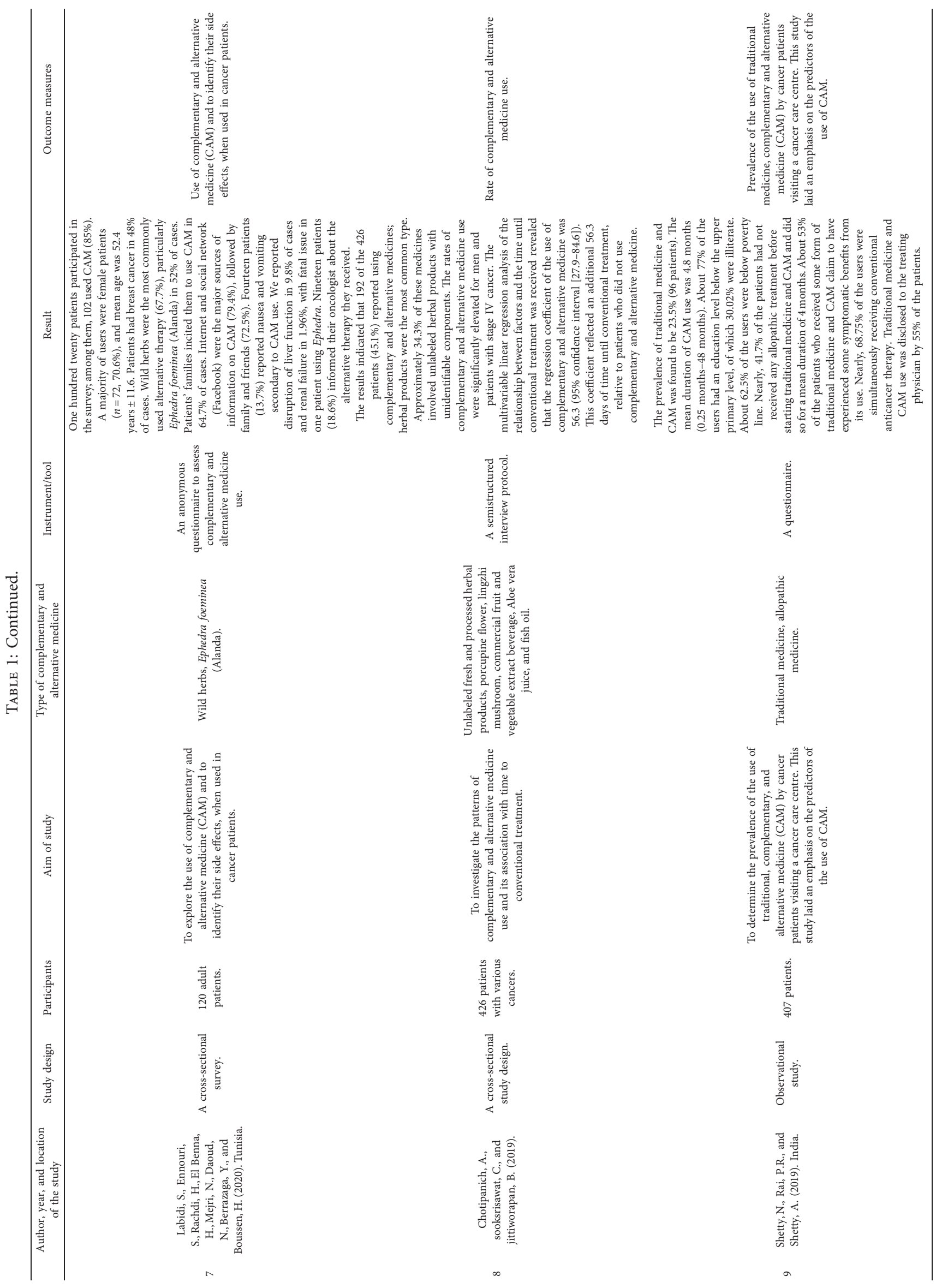




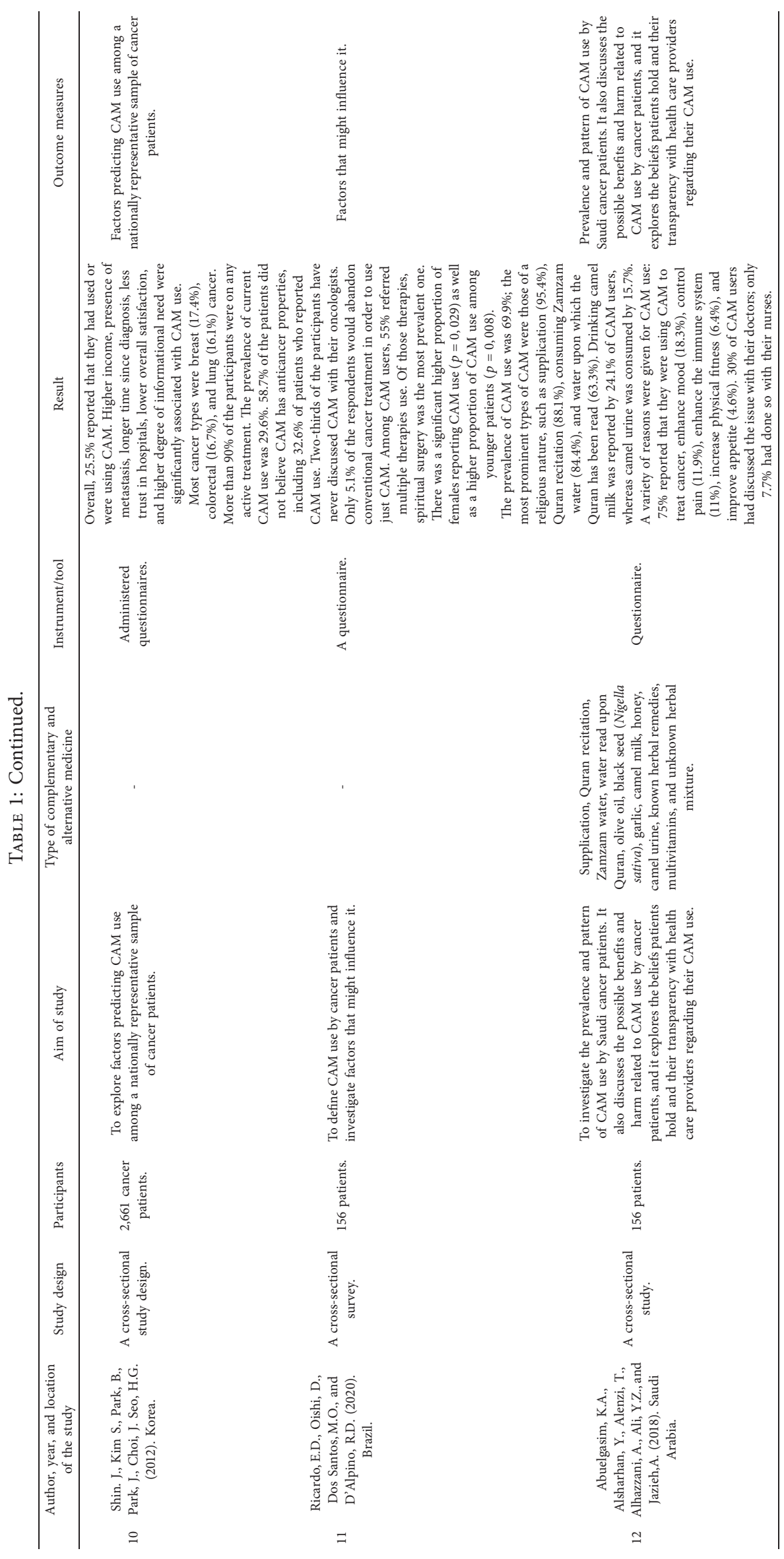




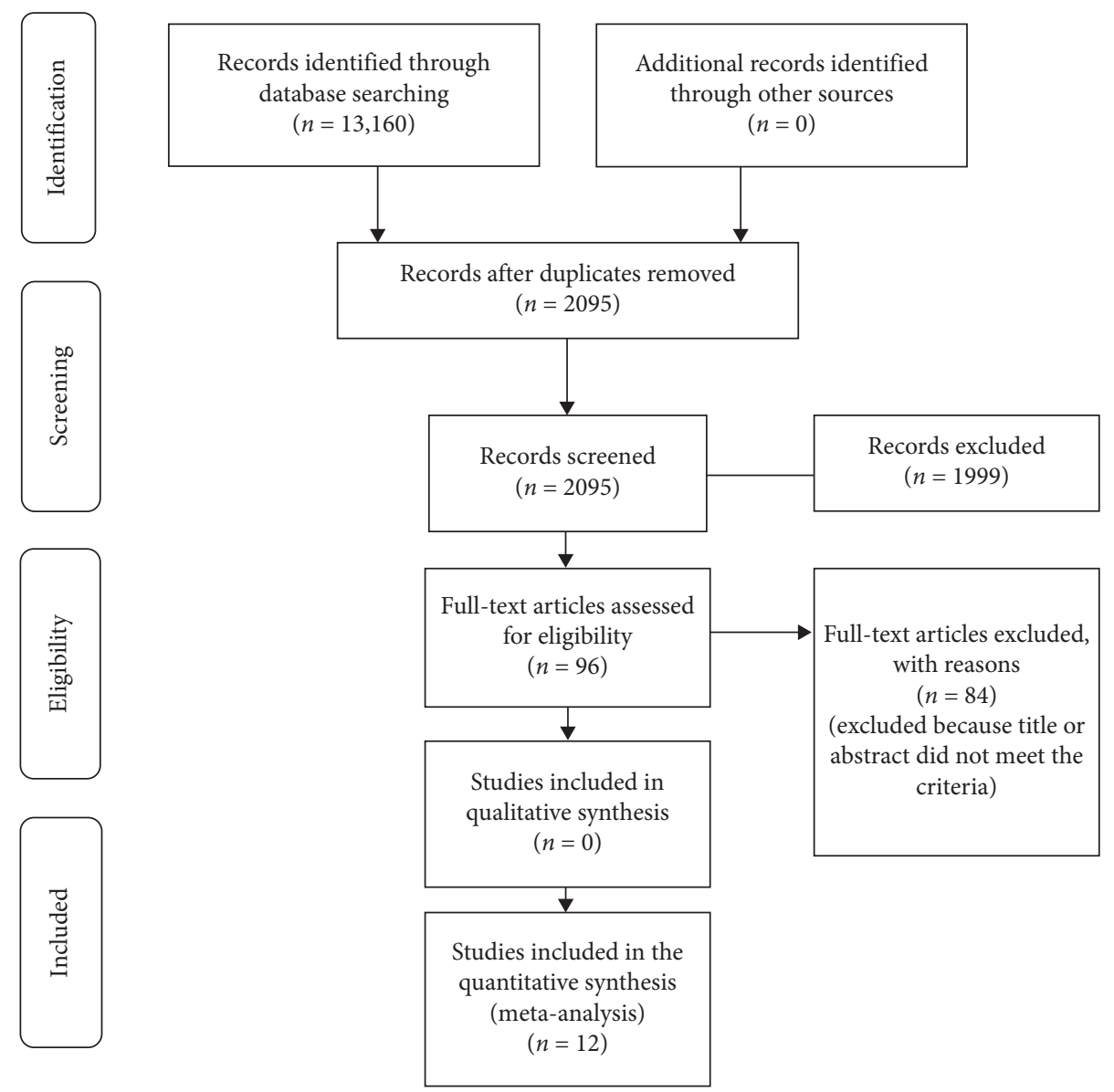

Figure 1: Flow diagram of the different phases of the systematic review.

most reliable source of information about complementary medicines was reported to be physicians $[4,5,15]$. Three studies reported that cancer patients discussed their CAM use with cancer care providers/physicians and nurses $[5,9,11,13,15]$.

\section{Discussion}

Herein, it was found that approximately $25 \%-80 \%$ of patients with cancer in the included 12 studies testified to the use of CAMs for the management of their cancer. The high prevalence of CAM use has been reported in recently published studies on CAM use among patients with different kinds of cancers $[17,18]$.

In this systematic review, the researcher found that patients with cancer practiced different types of CAMs, such as natural products, dietary supplements, prayers, and vitamins $[1,2,4,7,16]$. However, none of the studies mentioned the patients' perceptions regarding how well the CAMs they used worked in terms of improving their health. However, a few of the studies assessed patients' satisfaction of using CAMs [5, 9, 11, 13, 15].

Most of the patients did not discuss the use of CAMs to their healthcare providers $[1,9,11-15]$. The motivation for nondisclosure covered fear of a terrible reaction by their healthcare specialists, not being addressed regarding CAMs, and the perceived low knowledge of healthcare providers on CAMs, and thus there being no necessity to explore the topic with them $[17,18]$.

Patients, regardless of the type of cancer they had, practiced and used CAMs for numerous reasons and held various expectations, consisting of a cure for their cancer, control of most of their cancer-related symptoms, improving their immune system, and enhancing their physical and psychological well-being. The use of CAMs is hence likely to endure alongside standardized conventional cancer management and treatment, primarily because it has long been part of the culture of individuals and patients may thus trust CAM providers, and because of the convenient strategies and expense of CAMs [4, 5, 13, 15]. However, some other studies have reported that patients do not believe complementary and alternative medicines have an anticancer effect $[12,14]$.

Some of the other reviewed studies also showed that patients who use CAMs do so due to their dissatisfaction of conventional treatments, to the numerous side effects of standardized cancer drugs, and to their fear of surgery experiences, in addition to the fact that CAMs are more easily accessible and are less expensive than conventional treatments $[2,5,7,9,11,16]$. 


\section{Conclusions}

The majority of patients with cancer in the reviewed studies used various different types of CAMs concurrently with their cancer treatment. The use of CAMs in cancer patients can be inferred as an attempt to discover all potential options, an illustration of a coping style, or unmet desires in their current cancer care trajectory. In every cancer case, there should be adequate communication between patients and their healthcare providers, which is crucial for establishing physician-patient relationship trust.

\section{Abbreviations}

CAM: Complementary and alternative medicines.

\section{Data Availability}

No data were used to support this study.

\section{Conflicts of Interest}

The author declares that there are no conflicts of interest.

\section{Authors' Contributions}

F. Alsharif carried out the literature search, quality appraisal, and summation of results and approved the final manuscript.

\section{References}

[1] C. A. Buckner, R. M. Lafrenie, J. A. Dénommée, J. M. Caswell, and D. A. Want, "Complementary and alternative medicine use in patients before and after a cancer diagnosis," Current Oncology, vol. 25, no. 4, pp. e275-e281, 2018.

[2] World Health Organization (WHO), 2018, https://www.who.int/ news-oom/fact-heets/detail/cancer.

[3] K. Monicah, G. Mbugua, and R. Mburugu, "Use of complementary and alternative medicine among cancer patients in Meru County, Kenya," International Journal Of Professional Practice, vol. 7, no. 1, pp. 24-33, 2019.

[4] A. D. Mwaka, C. Abbo, and A. A. Kinengyere, "Traditional and complementary medicine use among adult cancer patients undergoing conventional treatment in sub-saharan africa: a scoping review on the use, safety and risks," Cancer Management and Research, vol. 12, pp. 3699-3712, 2020.

[5] L. G. Balneaves, M. E. Wong, A. J. Porcino, A. J. Porcino, T. L. O. Truant, S. E. Thorne et al., "Complementary and alternative medicine (CAM) information and support needs of Chinese-speaking cancer patients," Supportive Care in Cancer, vol. 26, no. 12, pp. 4151-4159, 2018.

[6] D. Polit and C. Beck, Essentials of Nursing Research: Appraising Evidence for Nursing Practice, Lippincott. W\& W, New York, 9th edition, 2018.

[7] K. Wode, R. Henriksson, L. Sharp, A. Stoltenberg, and J. H. Nordberg, "Cancer patients' use of complementary and alternative medicine in Sweden: a cross sectional study," BMC Complementary and Alternative Medicine, vol. 62, pp. 1-11, 2019.

[8] N. Shetty, P. R. Rai, and A. Shetty, "Study of the use of traditional, complementary, and alternative medicine in
Indian Cancer Patients," Indian Journal of Medical and Paediatrics Oncology, vol. 40, no. 3, pp. 365-368, 2019.

[9] A. M. Abdelmoaty, T. T. Amin, H. A. Obaid, O. A. A. Abdelsadek, U. H. B. Hassan et al., "Complementary medicines among Egyptian oncology patients at a tertiary level of care: pattern and motives," Health Society Journal, vol. 12, no. 2, p. $561,2018$.

[10] F. Amirmoezi, M. Araghizadeh, Z. Mohebbinia, R. Kamfiroozi, S. Haghpanah et al., "Use of complementary and alternative medicine among Iranian cancer patients in South of Iran," International Journal of Cancer Management, vol. 10, no. 10, Article ID e7233, 2017.

[11] R. M. Tappen, Advanced Nursing Research: From Theory to Practice, Jones \& Bartlett Learning, Burlington, MS, 2nd edition, 2016

[12] J. Shin, S. Kim, B. Park, J. Park, J. Choi, and H. G. Seo, "Predictors of Complementary and Alternative Medicine Use in Cancer Care: Results of a Nationwide Multicenter Survey in Korea," Evidence-based Complementary and Alternative Medicine, vol. 2012, p. 9, 2012.

[13] K. A. Abuelgasim, Y. Alsharhan, T. Alenzi, A. Alhazzani, Y. Z. Ali, and A. Jazieh, "The use of complementary and alternative medicine by patients with cancer: a cross-sectional survey in Saudi Arabia," BMC Complementary and Alternative Medicine, vol. 18, no. 1, pp. 1-8, 2018.

[14] K. Kessel, S. Lettner, C. Kessel, B. Henning, B. Tilo et al., "Use of complementary and alternative medicine as part of the oncological treatment: survey about patients' attitude towards CAM in a University-Based Oncology Center in Germany," PLoS One, vol. 11, Article ID e0165801, 2016.

[15] A. Chotipanich, C. Sooksrisawat, and B. Jittiworapan, "Association between complementary and alternative medicine use and prolonged time to conventional treatment among Thai cancer patients in a tertiary-care hospital," Peer Journal, vol. 7, Article ID e7159, pp. 1-11, 2019.

[16] M. Gras, A. Vallard, C. Brosse et al., "Use of complementary and alternative medicines among cancer patients: a singlecenter study," Oncology, vol. 97, no. 1, pp. 18-25, 2019.

[17] E. D. Ricardo, D. Oishi, M. O. Dos Santos, and R. D. D’Alpino, "Complementary and alternative medicine use among cancer patients at a private hospital in Brazil," Journal of Clinical Oncology, vol. 38, no. 15, pp. 209-214, 2020.

[18] S. Labidi, S. Ennouri, H. Rachdi et al., "Use of complementary and alternative medicine in cancer: a Tunisian single-center experience," Bulletin du Cancer, vol. 107, no. 2, pp. 209-214, 2020. 